\title{
Multi-Objective Optimal Design of a NEMA Design D Three-phase Induction Machine Utilizing Gaussian-MOPSO Algorithm
}

\author{
Dianhai Zhang*, Ziyan Ren* and Chang-Seop Koh ${ }^{\dagger}$
}

\begin{abstract}
This paper presents a multi-objective optimization approach to design rotor slot geometry of three-phase squirrel cage induction machine to achieve NEMA design D torque-speed (T-S) characteristics with high efficiency. The multi-objective Particle Swarm Optimization (MOPSO) algorithm combined with the adaptive response surface method and Latin hypercube sampling strategy is applied to obtain the Pareto optimal designs. In order to demonstrate the validity of the suggested optimal algorithm, an application to rotor slot design of three-phase induction motor is presented.
\end{abstract}

Keywords: High efficiency, Multi-Objective Optimization approach, NEMA design D machine, Three-phase squirrel cage induction machine.

\section{Introduction}

For the application involving pulsating inertia loads and frequent starting, the induction machine with high starting torque and high full load efficiency is essential. The torque-speed characteristics of the driving motor are important. The motor must have sufficient locked-rotor torque for initial starting. Typically, for these applications, an induction machine with NEMA design D torque-speed characteristics and high efficiency is in great demand [1].

NEMA design $\mathrm{D}$ induction machines, in generally speaking, are characterized by high starting torque, low starting current, and high operating slip. Full load slip for this kind of machines is quite high because of the high rotor resistance.

It is possible to produce a large-variety of torque-speed characteristics by varying the rotor geometry of induction machines. For achieving the torque-speed characteristics of the NEMA design D motor, an optimal shape design of the rotor slot is considered as a good choice [2].

Depending on the application, an induction machine designer is obliged to choose between high starting torque and high full load efficiency, by selecting the appropriate rotor bar resistance. In the foregoing researches, the rotor slot has been often optimized for general-purpose application to increase the starting torque [3-4] or efficiency at specific speed [5] based on equivalent circuit and finite element method.

However, a systematic optimal method to design the shape of rotor slot for specific torque-speed characteristics such as NEMA design D motor with high efficiency is quite difficult to find.

$\dagger$ Corresponding Author: College of Electrical and Computer Engineering, Chungbuk National University, Korea. (kohcs@chungbuk.ac.kr)

* School of Electrical Engineering, Shenyang University of Technology, China. (zhangdh@chungbuk.ac.kr)

Received: January 17, 2013; Accepted: August 12, 2013
In this paper, a systematic design method which determines the shape of rotor slot for a NEMA design D torque-speed characteristics with high full load efficiency is developed utilizing the multi-objective particle swarm optimization algorithm combined with an adaptive response surface method and Latin hypercube sampling strategy. The torque-speed characteristics and efficiency is evaluated by using the equivalent circuit method combined with the FEM.

\section{Calculation of Torque-speed Characteristics and Efficiency of Three-phase Induction Machine}

In this paper, the torque-speed characteristics and efficiency characteristics of a three-phase induction machine are analyzed by combining the equivalent circuit method (ECM) with the transient finite element method (FEM) [6-9].

\subsection{Calculation of torque-speed characteristics}

In order to achieve the design $\mathrm{D}$ torque-speed characteristics, it is necessary to evaluate not only the starting torque but also the maximum torque slip.

Fig. 1 shows a phase equivalent circuit of a three-phase induction machine, where $V_{1}$ is phase voltage, $R_{1}, X_{1}, X_{\mathrm{m}}$,

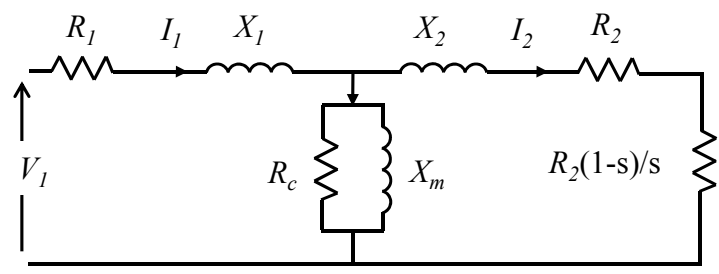

Fig. 1. A phase equivalent circuit of three-phase induction machine. 
and $I_{1}$ are the stator winding resistance, leakage reactance, magnetizing reactance, and current, respectively, and $R_{2}, X_{2}$, and $I_{2}$ are scaled rotor conducting bar resistance, reactance and rotor current, respectively, $s$ is slip of the rotor.

From the equivalent circuit of induction machine, the mechanical torque can be derived from power loss on $R_{2}$ $(1-\mathrm{s}) / \mathrm{s}$ at the specific slips shown as follows:

$$
T_{\text {mech }}=\frac{1}{\omega_{s}}\left\{\frac{3 V_{1 e q}^{2}\left(R_{2} / s\right)}{\left[R_{1 e q}+\left(R_{2} / s\right)\right]^{2}+\left(X_{1 e q}+X_{2}\right)^{2}}\right\}
$$

where $\omega_{\mathrm{s}}$ is synchronous speed, $V_{\text {leq }}$ and $Z_{\text {leq }}$ are defined as follows:

$$
\begin{gathered}
V_{\text {leq }}=\left|\frac{j R_{c} X_{m} \bar{V}_{1}}{\left(R_{1}+j X_{1}\right)\left(R_{c}+j X m\right)+j R_{c} X_{m}}\right| \\
Z_{\text {leq }}=\frac{j R_{c} X_{m}\left(R_{1}+j X_{1}\right)}{\left(R_{1}+j X_{1}\right)\left(R_{c}+j X_{m}\right)+j R_{c} X_{m}}
\end{gathered}
$$

where $\bar{V}_{1}$ is phasor representation of phase voltage.

To arrange the Eq. (1), the expression of mechanical torque at slip $s$ is expressed by using symbol $A, B$, and $C$ shown as follows:

$$
T_{\text {mech }}=\frac{1}{A s+B s^{-1}+C}
$$

where the coefficients $A, B$, and $C$ are defined as follows:

$$
\begin{gathered}
A=\frac{R_{c}^{2}\left[\left(X_{1}+X_{2}\right)^{2}+R_{1}^{2}\right]+X_{2}^{2}\left(R_{1}^{2}+X_{1}^{2}+2 R_{1} R_{c}\right)}{3 R_{2} V_{1}^{2} R_{c}^{2}} \\
+2 X_{2} \frac{X_{1}^{2}+R_{1}^{2}+X_{1} X_{2}}{3 R_{2} V_{1}^{2} X_{m}}+X_{2}^{2} \frac{X_{1}^{2}+R_{1}^{2}}{3 R_{2} V_{1}^{2} X_{m}^{2}} \\
B=\frac{R_{2}\left[\left(R_{1}+R_{c}\right)^{2}+X_{1}^{2}\right]}{3 V_{1}^{2} R_{c}^{2}}+\frac{2 R_{1} X_{1}}{3 V_{1}^{2} X_{m}}+R_{2} \frac{X_{1}^{2}+R_{1}^{2}}{3 V_{1}^{2} X_{m}^{2}} \\
C=\frac{2\left(R_{1}^{2}+X_{1}^{2}+R_{1} R_{c}\right)}{3 R_{c} V_{1}^{2}} .
\end{gathered}
$$

In general, the iron loss resistance and magnetizing reactance are much larger than other equivalent circuit parameters. Therefore, by assuming a large $R_{\mathrm{c}}$ and $X_{\mathrm{m}}$, the above equations can be simplified as follows:

$$
\begin{gathered}
A \approx \frac{\left[\left(X_{1}+X_{2}\right)^{2}+R_{1}^{2}\right]}{3 R_{2} V_{1}^{2}} \\
B \approx \frac{R_{2}}{3 V_{1}^{2}}
\end{gathered}
$$

$$
C=\frac{2 R_{1}}{3 V_{1}^{2}}
$$

From the simplified equations, the following equivalent circuit parameters are obtained:

$$
\begin{gathered}
R_{1}=\frac{3 C V_{1}^{2}}{2} \\
R_{2}=3 B V_{1}^{2} \\
X_{1}+X_{2} \approx \frac{3}{2} V_{1}^{2} \sqrt{4 A B-C^{2}} .
\end{gathered}
$$

By solving the parameters $A, B$, and $C$ from FEM results, the above equivalent circuit parameters are determined.

The mechanical torque expression also can be expressed in reciprocal way shown as follows:

$$
\tau_{\text {mech }}=\frac{1}{T_{\text {mech }}}=A s+B s^{-1}+C
$$

If there are three different torques and speed values, the mechanical torque expression can be written by matrix form shown as follows:

$$
\left[\begin{array}{c}
\tau_{\text {mech } 1} \\
\tau_{\text {mech } 2} \\
\tau_{\text {mech } 3}
\end{array}\right]=\left[\begin{array}{cc}
s_{1} & s_{1}^{-1} 1 \\
s_{2} & s_{2}^{-1} 1 \\
s_{3} & s_{3}^{-1} 1
\end{array}\right]\left[\begin{array}{l}
A \\
B \\
C
\end{array}\right] .
$$

By solving Eq. (8), the coefficients $A, B$, and $C$ can be expressed by three slips and torques values, respectively. The determinant of the coefficient matrix in (8) is nonzero and finite if and only if the three different torque values at asynchronous speeds. With given three different torque values, the torque-speed characteristics can be obtained from (7). The three torque-speed values can be calculated from the transient finite element method with constant speed. By using these three values, the entire torque-speed characteristics can be determined accurately. For example, the locked-rotor torque, full load torque, and an arbitrary asynchronous torque point are selected to evaluate. This method can prevent inaccuracy while only the equivalent circuit method is used.

Furthermore, the maximum torque slip, $s_{\max }$, can be derived from (7) and be expressed as follows:

$$
S_{\max }=\sqrt{B / A} .
$$

When the stator geometry of the machine is fixed, the torque-speed characteristics are related only with the geometry of the rotor slot.

Even for the parameters of stator side such as stator leakage inductance and magnetizing inductance are related 
with geometry of rotor slot opening which is considered by using Carter coefficient.

So it is possible to regulate maximum torque slip to close to one by varying the rotor bar geometry. The maximum starting torque, furthermore, also has relation with the rotor bar geometry.

The NEMA design D torque-speed characteristics can be achieved by limiting the maximum torque slip close to one, and maximizing the starting torque simultaneously.

\subsection{Calculation of efficiency}

For calculation of efficiency, the iron loss resistance has to be included in equivalent circuit. The iron loss resistance is determined from total iron loss of the induction machine [10]. The total iron loss $P_{\mathrm{c}}$ can be determined by integrating the losses over all the elements in the core. This involves calculating the magnetic flux density in each element from the vector potential solution of the FEM. The iron loss in each element is estimated by using the typical Steinmetz Eq. [11]. The iron loss resistance is derived as follows:

$$
R_{c}=\frac{3 E_{1}^{2}}{P_{c}}
$$

where $E_{1}$ is $r m s$ value of phase winding back EMF, and $P_{\mathrm{c}}$ is total iron loss. Other equivalent circuit parameters can be derived with different rotor slot geometries by using the analytical method. The efficiency $(\eta)$ is defined as the ratio of output mechanical power $\left(P_{o}\right)$ and input electric power $\left(P_{i}\right)$ calculated as follows:

$$
\begin{gathered}
P_{i}=3 V_{1} I_{1} \cos \varphi \\
P_{o}=3 I_{2}^{2}(1-s) R_{2} / s
\end{gathered}
$$

where $V_{1}$ and $I_{1}$ are phase voltage and current; $I_{2}$ and $R_{2}$ are current and scaled resistance of rotor conducting bar, respectively.

\subsection{Performance evaluation utilizing FEM and ECM}

In order to make a balance between calculation accuracy and computing cost, a hybrid method which combines the FEM and ECM is introduced to evaluate the starting torque, the maximum torque slip, and efficiency.

In section 2.1 and 2.2, the equivalent circuit equation of the induction machine is derived and then the maximum torque slip and efficiency can be calculated.

By using the adaptive response surface method, only the performance of the design on the sampling points needs to be determined by proposed method. For each specific design the evaluation flow is summarized as follows.

Step 1: The specific design is analyzed with transient FEM at the locked rotor state. After the steady state is reached for the analysis, the steady state torque can be calculated utilizing the average of time-torque curve for several periods. The starting torque is determined.

Step 2: For the previous specific design, the same transient FEM is applied with the rated speed and an arbitrary speed. After the calculation, the two torque-speed points can be determined;

Step 3: From Steps 1 and 2, totally three torque-speed points are determined by FEM. By using the method introduced in section 2.1 the parameters $A$, $B$, and $C$ can be determined from (8). The maximum torque slip is determined from (9).

Step 4: According to the calculation results from step 3, the iron loss under the rated condition can be determined by using the method which is introduced in section 2.2.

Step 5: Based on the result from step 4, the iron loss resistance $R_{\mathrm{c}}$ can be determined from (10).

Step 6: The stator winding resistance $R_{1}$, stator leakage reactance $X_{1}$ and magnetizing reactance $X_{\mathrm{m}}$ are determined from analytical method which is introduced in [6].

Step 7: For the calculation of rotor resistance $R_{2}$ and leakage reactance $X_{2}$, the parameter estimation method which is introduced in section 2.1 is applied. Up to now, the all equivalent circuit parameters are determined.

Step 8: The efficiency can be calculated by using (11) and (12) from the ECM.

Step 9: The starting torque, maximum torque slip, and efficiency are determined for each sampling design by repeating the step 1 to step 8 .

\section{Optimal Design Method of Rotor Slot}

The design $\mathrm{D}$ torque-speed characteristics can be obtained by controlling the maximum torque slip close to one and maximizing starting torque simultaneously. In addition, the maximum full load efficiency should be achieved at the same time. To satisfy this requirement, a three-objective optimization problem with design variable vector $\mathbf{x}$ can be formulated as follows:

$$
\begin{array}{ll}
\text { Maximize } & f_{1}(\mathbf{x})=T_{s} \\
\text { Minimize } & f_{2}(\mathbf{x})=|1-\sqrt{B / A}| . \\
\text { Maximize } & f_{3}(\mathbf{x})=\eta \\
\text { subject to } & \mathbf{x}_{L} \leq \mathbf{x} \leq \mathbf{x}_{U}
\end{array}
$$

Model (13) appears straightforward and profitable to satisfy the design requirement. However, a perfect multiobjective optimization algorithm is prerequisite for good solution. 
In order to reduce the influence of performance of multiobjective optimization algorithm, model (13) can be simplified into a bi-objective optimization problem utilizing weighting factor $(0 \leq \alpha \leq 1)$ shown as:

$$
\begin{array}{ll}
\text { Minimize } & f_{1}(\mathbf{x})=\alpha|1-\sqrt{B / A}|-(1-\alpha) T_{s} \\
\text { Maximize } & f_{2}(\mathbf{x})=\eta \\
\text { subject to } & \mathbf{x}_{L} \leq \mathbf{x} \leq \mathbf{x}_{U} .
\end{array}
$$

For the sake of accuracy of torque and iron loss calculation of induction machine, the transient FEM is required. In order to overcome the problem of expensive computation cost of optimal design, an adaptive response surface method with an adaptive Latin hypercube sampling strategy has been applied to rotor slot optimal design [12].

Gaussian-MOPSO algorithm is applied to solve model (14) to get the proper Pareto-front from which a designer may select an optimal design based on different requirement [13].

The Gaussian-MOPSO is developed by using social and cognitive time-variant factors, where the velocity of particles is updated based on a truncated Gaussian distribution. The Pareto front from Gaussian-MOPSO algorithm is proved to be uniformly spaced than other counterparts, and be closer to the utopia point.

The detail optimization algorithm developed in this paper is shown in Fig. 2.

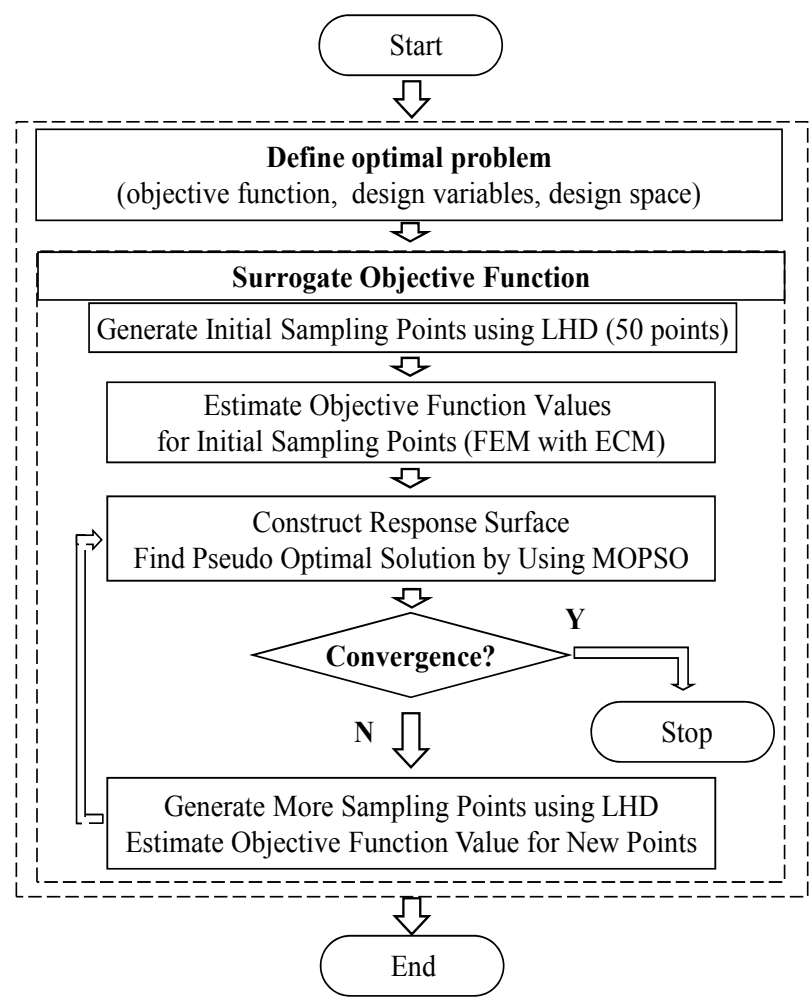

Fig. 2. Flow chart of optimal design algorithm.

\section{Optimal Results}

In order to validate the suggested optimal design method, a general purpose three-phase induction machine, shown in Table 1 and Fig. 3, is used.

There are several kinds of candidate initial rotor slot shape to be adopted. For the purpose of simplicity, a circular rotor slot is selected without loss of generality.

In the circular slot, the main degrees of freedom characterizing the shape are width $(w)$, height of rotor slot opening $(h)$, and radius of rotor conducing bar $(r)$, respectively, of which design range is listed in Table 2.

Pareto-optimal solutions of (14) with $\alpha=0.5$ are found from response surface by using MOPSO are shown in Fig. 4. For example, the designer can select solution $B$ as the optimal design. The variation of rotor slot shape for initial and optimal design is shown in Fig. 5. Obviously, the rotor

Table 1. Specification of the induction motor

\begin{tabular}{|c|c|}
\hline Item & Value \\
\hline Power $(\mathrm{kW})$ & 3.7 \\
\hline Phase voltage $(\mathrm{V})$ & 380 \\
\hline Frequency $(\mathrm{Hz})$ & 50 \\
\hline Number of poles & 8 \\
\hline Number of slots of stator/ rotor & $48 / 44$ \\
\hline$R_{1}(\Omega)$ & 2.6864 \\
\hline
\end{tabular}

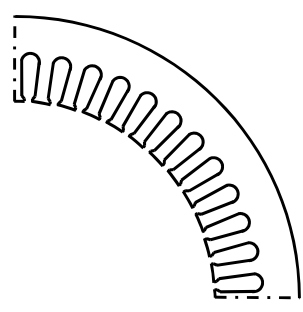

(a) stator

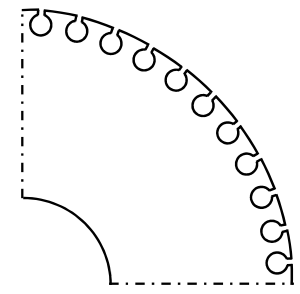

(b) rotor
Fig. 3. Stator and rotor of the three-phase induction motor.

Table 2. Design variables for initial and optimal design

\begin{tabular}{c|c|c|c}
\hline Designs & $W[\mathrm{~mm}]$ & $H[\mathrm{~mm}]$ & $R[\mathrm{~mm}]$ \\
\hline Initial design & 1.0 & 0.5 & 2.5 \\
\hline Minimum & 0.5 & 0.3 & 1.0 \\
\hline Maximum & 4.0 & 1.5 & 3.0 \\
\hline Optimal design & 2.975 & 0.32 & 1.61 \\
\hline
\end{tabular}

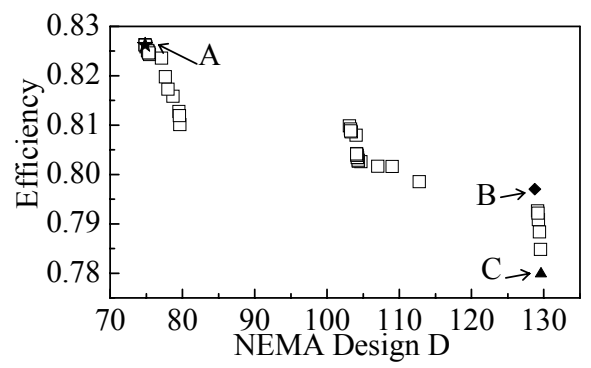

Fig. 4. Pareto-optimal designs. 


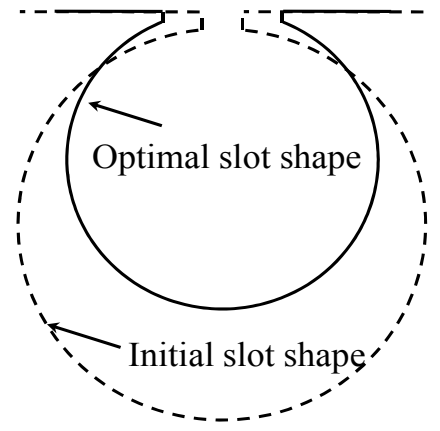

Fig. 5. Variation of circular rotor slot shape.

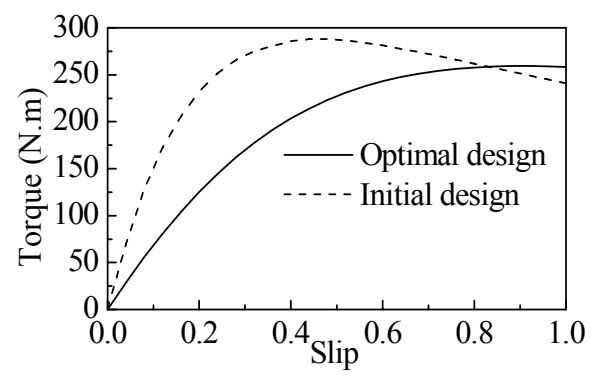

Fig. 6. Comparison of torque-speed curves.

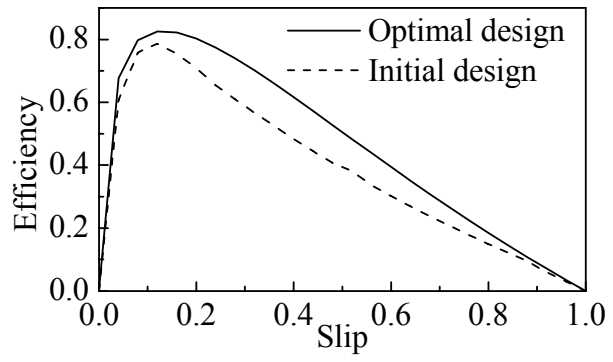

Fig. 7. Comparison of efficiency curves.

slot becomes smaller than the initial design because of the bigger rotor resistance requirement and the slot opening becomes wider and shallower than initial design because of lower rotor slot leakage reactance requirement. Table 2 shows the geometry of design variables for initial, optimal design, and design space. The corresponding torque-speed characteristics for initial and optimal designs are shown in Fig. 6. From the curves, it is found that the optimal design has a higher starting torque and more close to design D torque-speed characteristics than the initial design. Fig. 7 compares the efficiency characteristics for optimal design and initial design. The efficiency at $\mathrm{s} \approx 0.08$ (full load slip) of optimal design is higher than initial rotor slot shape design.

However, the design $B$ is not an only design selection; other designs also can be selected as candidate designs. For example, in Pareto-optimal solutions, the design $A$ or $C$ also can be treated as optimal designs. Fig. 8 shows the torque-speed and efficiency characteristics for design $A$. It can be seen that this design took more effort on high efficiency instead of NEMA design D torque-speed

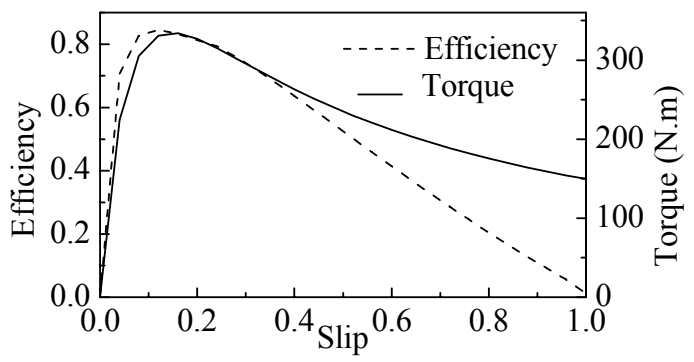

Fig. 8. Torque-speed and efficiency characteristics for design A.

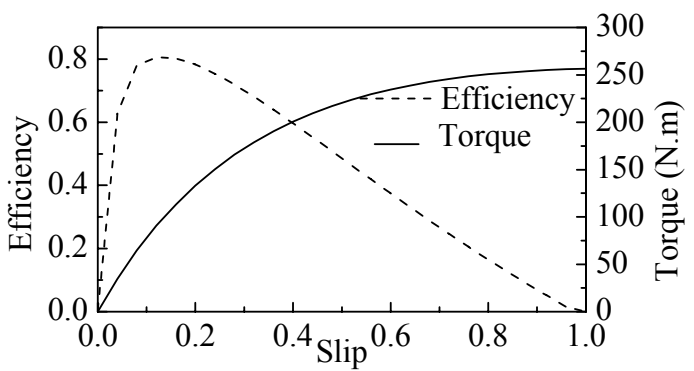

Fig. 9. Torque-speed and efficiency characteristics for design $\mathrm{C}$.

characteristics. Conversely, the design $C$ takes more effort on NEMA design D torque-speed characteristics than other designs shown as Fig. 9. In the real world, the designer may select one of the candidate designs as optimal design depending on the real application.

\section{Conclusion}

The optimal design algorithm, which can obtain the NEMA design D torque-speed characteristics with high full load efficiency, is presented. The developed algorithm is applied to the rotor slot shape optimal design for threephase induction machine. Through design applications with the sample rotor slot, the developed optimal design method is demonstrated to give an optimal design for a desired torque-speed characteristics and high full load efficiency.

\section{Acknowledgements}

This work was supported by the Human Resources Development of the KETEP grant funded by the Korea government Ministry of Knowledge Economy (No. 20104010100600).

\section{References}

[1] Energy management guide for selection and use of fixed frequency medium AC squirrel-cage poly-phase 
induction motors. NEMA standards publication MG 10-2001, NEMA.

[2] D. H. Zhang, C. S. Park, and C. S. Koh, "A new optimal design method of rotor slot of three-phase squirrel cage induction motor for NEMA class D speed-torque characteristics using Multi-objective optimization algorithm," IEEE Trans. Magn., vol.48, no.2, pp.879-882, Feb. 2012.

[3] J. Appelbaum, "Determination of slot dimensions of double squirrel-cage and deep-bar rotors," IEEE Trans. on Power Apparatus and Systems, pp.15131518, 2007.

[4] J. P. Wieczorek, Ö. Göl, and Z. Michalewicz, “An evolutionary algorithm for the optimal design of induction motors," IEEE Trans. Magn., vol.34, no.6, pp.3882-3887, Nov. 1998.

[5] P. W. Han, U. J. Seo, J. H. Choi, Y. D. Chun, D. H. Koo, and J. Lee, "Optimizing design variables for high efficiency induction motor considering cost effect by using genetic algorithm," Journal of Electrical Engineering \& Technology., vol.7, no.1, pp.46-50, 2012.

[6] A. Boglietti, A. Cavagnino and M. Lazzari, "Computational algorithms for induction motor equivalent circuit parameter determination part I: resistances and leakage reactances," IEEE Trans. on Industrial Electronics., no.99, 2010.

[7] S. Jangjit and P. Laohachai, "Parameter estimation of three-phase induction motor by using genetic algorithm," Journal of Electrical Engineering \& Technology., vol.4, no.3, pp.360-364, 2009.

[8] S. R. Nelatury, "Uniqueness of torque-speed characteristics of an induction motor," IEEE Trans. Magn., vol.40, no.5, pp.3431-3433, Sep. 2004.

[9] P. Zhou, J. Gilmore, Z. Badics, and Z. J. Cendes, "Finite element analysis of induction motors based on computing detailed equivalent circuit parameters," IEEE Trans. Magn., vol.34, no.5, pp.3499-3502, Sep. 1998.

[10] W. N. Fu, P. Zhou, D. Lin, S. Stanton, and Z. J. Cendes, "Modeling of solid conductors in twodimensional transient finite-element analysis and its application to electric machines," IEEE Trans. Magn., vol.40, no.2, pp.426-434, Mar. 2004.

[11] J. J. Lee, Y. K. Kim, H. Nam, K. H. Ha, J. P. Hong, and D. H. Hwang, "Loss distribution of three-phase induction motor fed by pulse-width-modulated inverter," IEEE Trans. Magn., vol. 40, no. 2, pp. 762765, Mar. 2004.

[12] H. S. Yoon, Y. H. Eum, Y. L. Zhang, and C. S. Koh, "Multi-objective optimal design of a single phase AC solenoid actuator used for maximum holding force and minimum eddy current loss," Journal of Electrical Engineering \& Technology., vol.3, no.2, pp. 218-223, 2008.

[13] L. S. Coelho, H. V. Ayala, and P. Alotto, "A multi- objective Gaussian particle swarm approach applied to electromagnetic optimization," IEEE Trans. Magn., vol.46, no.8, pp.3289-3292, Aug. 2010.

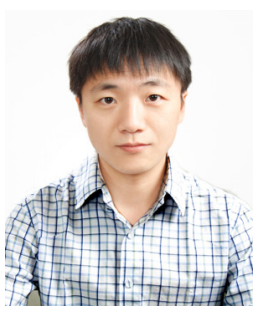

Dianhai Zhang He received his B.S. M.S., and Ph.D degrees in the biomedical engineering, electrical machine and electric apparatus from Shenyang University of Technology and electrical engineering from Chungbuk National University, in 2006, 2009 and 2013 , respectively. He is presently a lecturer at college of electrical engineering, Shenyang University of Technology, China. His research interests include design of electromagnetic devices and numerical analysis of electromagnetic fields. He can be contacted at zhangdh@chungbuk.ac.kr.

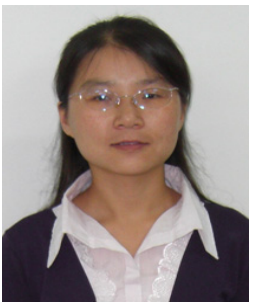

Ziyan Ren She received her B.S M.S., and $\mathrm{Ph} . \mathrm{D}$ degrees in the biomedical engineering, theory of electrical engineering and new technology from Shenyang University of Technology and electrical engineering from Chungbuk National University, in 2006, 2009 and 2013, respectively. She is currently working as Post-doctor at College of Electrical and Computer Engineering in Chungbuk National University, Korea. Her research interests include the optimal design of electromagnetic devices, the numerical analysis of electromagnetic fields. She can be contacted at renziyan@ chungbuk.ac.kr.

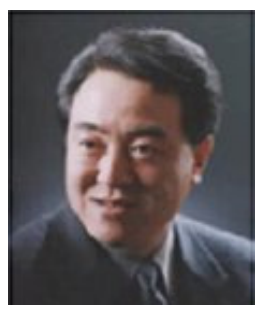

Chang-Seop Koh He received his B.S., M.S., and $\mathrm{Ph}$. D. degrees in electrical engineering from Seoul National University, Seoul, Korea, in 1982, 1986, and 1992, respectively. He was visiting Professor at the Department of Electrical and Computer Engineering, Florida International University, Florida, USA, and at the Department of Electrical and Computer Engineering, Texas A\&M University, Texas, USA, from May 1993 to April 1994, and from February 2003 to January 2004, respectively. He was also a Senior Researcher at the Central Research Institute of Samsung Electro-Mechanics Co., Ltd., from May 1994 to August 1996. He has been a Professor with the College of Electrical and Computer Engineering, Chungbuk National University, Korea, since 1996. His research interests include electric machine design, numerical analysis of electric machines using the finite element. He can be contacted atkohcs@chungbuk.ac.kr. 